\section{Validación de cuestionario autoaplicable para un índice de alimentación mediterránea en Chile}

\author{
GUADALUPE ECHEVERRÍA ${ }^{1, \mathrm{a}}$, INÉS URQUIAGA ${ }^{1, \mathrm{~b}}$, \\ MARÍA JOSÉ CONCHA ${ }^{1, \mathrm{c}}$, CATALINA DUSSAILLANT ${ }^{1, \mathrm{~d}}$, \\ LUIS VILLARROEL ${ }^{2, \mathrm{e}}$, NICOLÁS VELASCO ${ }^{3}$, \\ FEDERICO LEIGHTON ${ }^{1}$, ATTILIO RIGOTTI ${ }^{1,3, \mathrm{f}}$
}

\section{Validation of self-applicable questionnaire for a Mediterranean dietary index in Chile}

Background: Availability of brief dietary indexes that can effectively evaluate dietary patterns and their association with health is critical for prevention and management of several chronic disease conditions. Aim: To adapt a self-applicable Mediterranean Dietary Index in Chile (Chilean-MDI). Material and Methods: The Chilean-MDI was developed based on a previous Mediterranean eating score that was adapted to Chilean dietary habits. This index was further validated in a sample of 153 adults by comparing the concordance between the results obtained by self-application of the Chilean-MDI with those obtained by a trained nutritionist. Additionally, the index was applied in a sample of 53,366 Chilean adults in order to describe the diet quality of our population. Results: There was an adequate concordance between findings obtained by self-application of the Chilean-MDI and those achieved by the nutritionist. The application of the index in Chilean adult population showed a better diet quality (high Mediterranean diet adherence) among women, with advanced age and among people with higher educational levels. Conclusions: The Chilean-MDI can be successfully self-applied to portray the overall diet quality in the Chilean adult population. Additionally, this dietary index describes overall food intake in Chilean adults, showing demographic trends that are comparable to those obtained with similar indexes applied in other populations.

(Rev Med Chile 2016; 144: 1531-1543)

Key words: Chile; Diet, Mediterranean; Indexes; Nutrition Assessment.
${ }^{1}$ Centro de Nutrición Molecular y Enfermedades Crónicas. Escuela de Medicina, Pontificia Universidad Católica, Santiago, Chile.

2Departamento de Salud Pública. Escuela de Medicina, Pontificia Universidad Católica, Santiago, Chile.

${ }^{3}$ Departamento de Nutrición, Diabetes y Metabolismo,

Escuela de Medicina, Pontificia Universidad Católica, Santiago,

Chile.

Ingeniero Civil. MSc.

bBioquímica. PhD.

'Nutricionista. MSC.

${ }^{\mathrm{d} M S c}$.

eEstadístico. PhD.

${ }^{\mathrm{f}} \mathrm{PhD}$.

Fuente de apoyo financiero: Fundación Banmédica. Fundación Banmédica no tuvo influencia en el diseño del estudio; en la recolección de los datos, en el análisis o interpretación de los datos; ni en la preparación, revisión o aprobación del manuscrito.

Recibido el 1 de agosto de 2016, aceptado el 14 de noviembre de 2016.

Correspondencia a:

Dr. Attilio Rigotti

Departamento de Nutrición, Diabetes y Metabolismo, Escuela de Medicina, Pontificia Universidad Católica de Chile. Marcoleta 367, interior, $4^{\circ}$ Piso, Santiago, CP 8330024, Chile. Teléfono: +5622354 3862 arigotti@med.puc.cl
L a dieta es fundamental en la prevención y tratamiento de numerosas enfermedades crónicas. Sin embargo, la metodología que mide el impacto de la ingesta alimentaria en salud es compleja y representa un gran desafío en epidemiología nutricional. Dada la laboriosidad asociada a la aplicación de encuestas de alimentación detalladas, existen índices o scores más sencillos que permiten evaluar patrones globales de alimentación. Estos índices evalúan la dieta en forma integral, más allá del análisis del consumo de alimentos individuales, ya que el impacto de la dieta en la salud o enfermedad está probablemente determinado por el efecto sinérgico de múltiples alimentos ${ }^{1-4}$.

Así, existen múltiples scores de alimentación validados en su aplicabilidad y relación con outcomes de salud ${ }^{4,5}$. Recientemente, una cohorte multiétnica mostró que una dieta más saludable -medida mediante diferentes índices 
de alimentación- se asocia a menor mortalidad total e incidencia de enfermedad cardiovascular y cáncer ${ }^{6}$. Actualmente, los cuatro scores más ampliamente utilizados son el Healthy Eating Index (HEI) ${ }^{7}$, el Diet Quality Index (DQI) ${ }^{8}$, el Healthy Diet Indicator (HDI $)^{9}$ y el Mediterranean Diet Score $(\mathrm{MDS})^{10}$.

Recientemente, este último índice y otros similares ${ }^{11}$ han captado mucha atención por el efecto saludable de la dieta mediterránea. Esta alimentación es el patrón dietario con mayor evidencia sobre sus efectos positivos en salud humana, incluyendo menor riesgo de enfermedad cardiovascular, diabetes, cáncer, deterioro psicoorgánico y mortalidad total ${ }^{10-13}$. Varios índices permiten medir la adherencia a este patrón dietario como el MDS, el índice de adecuación mediterráneo ${ }^{14}$ y el índice SUN (Seguimiento Universidad de Navarra $)^{15}$, entre otros ${ }^{11}$. Utilizando estos índices, numerosos estudios transversales y de cohortes han correlacionado este patrón alimentario con menor incidencia y/o mortalidad de enfermedad cardiovascular, neoplásica, neurodegenerativa y mortalidad total ${ }^{10,16-19}$.

En nuestro país, Leighton y $\operatorname{cols}^{20}$ crearon un índice de alimentación mediterránea que fue aplicado en un estudio de intervención dietaria en trabajadores chilenos. Este índice demostró ser efectivo para la medición de la composición de la dieta de sujetos chilenos y cambios favorables en este indicador se correlacionaron con efectos positivos en salud ${ }^{20}$, como ha ocurrido con otros índices mediterráneos aplicados en estudios internacionales ${ }^{15,21}$.

En base a esta experiencia previa ${ }^{20}$, el presente estudio reporta un Îndice Chileno de Dieta Mediterránea (IDM-Chile), adaptado a la realidad y los hábitos alimentarios nacionales, que puede ser entendido y autocontestado por los usuarios -sin necesidad de apoyo de personal entrenadoy que permite evaluar la calidad de la dieta en la población adulta chilena de acuerdo al patrón de alimentación mediterránea.

\section{Materiales y Métodos}

\section{Diseño de un índice chileno de dieta mediterránea autoaplicable, IDM-Chile}

El IDM-Chile se desarrolló a partir del índice generado por Leighton y cols ${ }^{20}$. Este instrumento evalúa la frecuencia de consumo de 14 grupos de alimentos (Anexo 1). La cantidad consumida de cada grupo de alimentos determina valores de 0 , 0,5 y 1 punto, según la recomendación derivada de una dieta mediterránea (Tabla 1). La sumatoria de los valores de cada grupo alimentario genera un puntaje total, que puede variar entre 0 puntos -ausencia de adherencia- y 14 puntos -máxima adherencia- al patrón de dieta mediterránea. El algoritmo detallado de cálculo del puntaje del IDM-Chile se presenta en el Anexo 2.

\section{Muestra estudiada para validación de la auto- aplicabilidad del cuestionario de IDM-Chile}

La validación del IDM-Chile se realizó en una muestra de 153 adultos chilenos presumiblemente sanos, sin consejería previa sobre su estilo de alimentación ni participación en un programa de baja de peso dentro de los últimos 6 meses (48\% hombres, con edad promedio de 42,5 \pm 9,7 años, rango de edades entre 21 y 62 años, y $54 \%$ con más de 12 años de educación formal). Inicialmente, estos individuos autorrespondieron el cuestionario del IDM-Chile y posteriormente las respuestas fueron obtenidas mediante encuesta realizada por una nutricionista, analizando el grado de asociación y concordancia de las respuestas logradas en ambos casos para validar la autoaplicabilidad del mismo. Este estudio fue aprobado por el Comité de Ética en Investigación de la Facultad de Medicina de la Pontificia Universidad Católica de Chile.

\section{Autoaplicación online del IDM-Chile en adultos chilenos}

Desde el año 2010, el IDM-Chile ha estado disponible a través de la plataforma online del Programa Aliméntate Sano (www.alimentatesano. cl) de la Pontificia Universidad Católica de Chile y ha sido autoaplicado en 53.366 adultos chilenos, disponiendo de sus datos personales completos (Tabla 2).

Mediante la información obtenida de la autoaplicación del IDM-Chile en esta muestra, se evaluaron las características de la alimentación y su nivel de adherencia al patrón de dieta mediterránea según sexo, edad y nivel educacional. La categorización del nivel de adherencia a este patrón dietario se realizó arbitrariamente según terciles de puntaje. Así, un puntaje entre 9 y 14 puntos fue considerado como una dieta con alta adherencia a alimentación de tipo mediterráneo 
Tabla 1. Descripción de los componentes y criterios de puntuación del índice chileno de dieta mediterránea (IDM-Chile)

\begin{tabular}{|c|c|c|c|c|c|c|}
\hline \multirow[t]{2}{*}{ Grupos de alimentos } & \multirow{2}{*}{$\begin{array}{l}\text { Unidad de } \\
\text { medida }\end{array}$} & \multicolumn{5}{|c|}{ Puntaje } \\
\hline & & $\mathbf{0}$ & 0,5 & 1 & 1,5 & 2 \\
\hline 1. Verduras & Porciones/día & $<1$ & 1 a 3 & $\geq 3$ & - & - \\
\hline 2. Legumbres & Veces/semana & $<1$ & 1 a 2 & $>2$ & - & - \\
\hline 3. Frutos secos & Puñados/semana & $<1$ & 1 a 2 & $>2$ & - & - \\
\hline 4. Frutas & Porciones/día & $<1$ & 1 a 2 & $\geq 2$ & - & - \\
\hline 5. Cereales integrales & Porciones/día & $<1$ & 1 a 2 & $\geq 2$ & - & - \\
\hline $\begin{array}{l}\text { 6. Lácteos fermentados y } \\
\text { descremados }\end{array}$ & Tazas/día & No consume & $\leq 1$ & $>1$ & - & - \\
\hline 7. Lácteos enteros & Porciones/día & $\geq 1$ & $<1$ & No consume & - & - \\
\hline 8. Pescados y mariscos & Veces/semana & $<1$ & 1 a 2 & $>2$ & - & - \\
\hline $\begin{array}{l}\text { 9. Carnes blancas y rojas } \\
\text { magras }\end{array}$ & Veces/semana & $<20>8$ & 2 a 4 & 5 a 8 & - & - \\
\hline $\begin{array}{l}\text { 10. Carnes rojas grasas y } \\
\text { carnes procesadas }\end{array}$ & Veces/semana & $>2$ & 1 a 2 & $<1$ & - & - \\
\hline 11. Aceite de oliva & $\begin{array}{l}\text { Aceite de oliva (O): } \\
\text { cucharaditas/día }\end{array}$ & $<1$ & 1 a 2 & $\geq 3$ & - & - \\
\hline у & $\begin{array}{l}\text { Palta }(P) \text { : } \\
\text { unidades/semana }\end{array}$ & $<1 / 2$ & $1 / 2$ a 3 & $>3$ & - & - \\
\hline \multirow{2}{*}{$\begin{array}{l}\text { 12. Otras grasas saludables: } \\
\text { palta y aceite canola }\end{array}$} & $\begin{array}{l}\text { Aceite canola }(C) \text { : } \\
\text { tipo de consumo }\end{array}$ & No consume & ocasional & regular & - & - \\
\hline & & $\begin{array}{c}O=0 y \\
(P+C)<1\end{array}$ & $\begin{array}{c}O=0,5 y \\
(P+C)<1 \\
O \\
O=0 y \\
(P+C)=1\end{array}$ & $\begin{array}{c}O=0,5 y \\
(P+C)=1 \\
O \\
O=0 y \\
(P+C) \geq 1,5\end{array}$ & $\begin{array}{c}O=0,5 \\
y(P+C) \\
\geq 1,5\end{array}$ & $\mathrm{O}=1$ \\
\hline 13. Vino & Copas/día & $\begin{array}{l}\text { No consume } \\
\text { o consume } \\
<4 \text { días/sem }\end{array}$ & $\begin{array}{l}<1 \text { o }>2, y \\
\geq 4 \text { días } / \text { sem }\end{array}$ & $\begin{aligned} & 1 \text { a } 2, y \\
\geq & 4 \text { días } / \text { sem }\end{aligned}$ & - & - \\
\hline \multirow{2}{*}{$\begin{array}{l}\text { 14. Azúcar }(A) \text { y snacks y/o } \\
\text { bebidas azucarados }(A A)\end{array}$} & A: cucharaditas/día & $\geq 4$ & $<4$ & $<4$ & - & - \\
\hline & AA: porciones/día & $\begin{array}{l}\text { Consumo } \\
\text { diario de AA }\end{array}$ & $\begin{array}{l}\text { Consumo } \\
\text { diario de AA }\end{array}$ & $\begin{array}{l}\text { Consumo no } \\
\text { diario de AA }\end{array}$ & - & - \\
\hline
\end{tabular}

(buena calidad o saludable), entre 5 a 8,5 se categorizó como una dieta con adherencia moderada a alimentación mediterránea (regular calidad) y menos de 5 puntos se definió como una dieta con baja adherencia a esta dieta (mala calidad). Además, se evaluó la prevalencia del consumo saludable -según el patrón mediterráneo- de los diferentes alimentos considerados por este índice, ajustando los análisis por sexo, edad y región de residencia de la población chilena.

\section{Análisis estadístico}

Para validación de la autoaplicabilidad del IDM-Chile, se determinó el grado de asociación y concordancia entre las respuestas obtenidas del cuestionario autoaplicado (IDM-a) versus aquellas resultantes del IDM-Chile aplicado por una nutricionista (IDM-n), usando análisis de correlación de Spearman, test de Bland \& Altman, prueba t Student para muestras pareadas y valor de kappa para cada uno de los ítems del instrumento. 
Tabla 2. Características de la muestra chilena de adultos $>20$ años $(n=53.366)$ proveniente del Programa Aliméntate Sano

\begin{tabular}{|ll|}
\hline Sexo femenino (\%) & 65,5 \\
\hline Edad, años (media \pm desviación estándar) & $34,8 \pm 11,9$ \\
\hline Adultos jóvenes, 20 a 40 años (\%) & 72,1 \\
\hline Nivel educacional alto, con más de 12 años de escolaridad (\%) & 72,9 \\
\hline Residentes en Región Metropolitana (\%) & 57,6 \\
\hline Fumadores (\%) & 42,4 \\
\hline Actividad física: & 41,3 \\
\hline - Alta (\%) & 35,8 \\
\hline Bedia (\%) & 22,9 \\
\hline Índice de masa corporal, $\mathrm{kg} / \mathrm{m}^{2}$ (media \pm desviación estándar) & $26,1 \pm 4,6$ \\
\hline Sobrepeso y obesidad $(\%)$ & 53,7 \\
\hline
\end{tabular}

Adicionalmente, el test de Kolmogorov-Smirnov analizó la normalidad de la distribución de los puntajes derivados de la aplicación del instrumento en la muestra de validación.

Para el análisis de los resultados de la autoaplicación online del IDM-Chile en adultos chilenos usuarios del Programa Aliméntate Sano, las variables numéricas continuas se presentan como promedio e intervalo de confianza de $95 \%$ o como promedio y desviación estándar, mientras que las variables categóricas se presentan como número de casos y porcentajes. Se utilizó el test Chi-cuadrado para comparar porcentajes y el test t Student para comparación de valores promedios. Una diferencia se consideró estadísticamente significativa cuando el valor p resultó inferior a 5\%.

Todos los análisis estadísticos fueron realizados usando el programa estadístico SPSS v17.0 para Windows.

\section{Resultados}

Validación de la autoaplicabilidad del IDM-Chile en población adulta chilena

Aplicando la prueba de Kolmogorov-Smirnov, los puntajes totales obtenidos del IDM-Chile autoaplicado (IDM-a) o aplicado por una nutricionista (IDM-n) mostraron una distribución normal $(\mathrm{p}=0,164$ para IDM-a y $\mathrm{p}=0,111$ para DM-n) en la muestra de 153 adultos chilenos incluidos en esta validación.
El promedio de puntuación del IDM-a fue de $5,60 \pm 1,57$ puntos, con un rango de respuestas que varió entre los 2 y los 9 puntos. En este mismo grupo de individuos, el promedio de puntaje resultante del IDM-n fue levemente mayor, de $5,71 \pm 1,61$ puntos (rango entre 2 y 10 puntos), pero sin diferencia significativa $(\mathrm{p}>0,05$ con t Student para muestras pareadas). Además, la correlación entre ambas mediciones fue positiva y significativa, con un coeficiente de correlación de $0,94(\mathrm{p}<0,001$, Figura 1$)$.

La Figura 2 muestra el análisis de Bland y Altman que evaluó la concordancia entre los resultados de puntajes totales obtenidos para IDM-a versus IDM-n. Así, el promedio de las diferencias entre las puntuaciones derivadas de ambos instrumentos fue $0,14 \pm 0,56$ puntos. No se demostró un error sistemático, ya que los puntos se distribuyeron de forma aleatoria a uno y otro lado del eje de la abscisa correspondiente a ausencia de diferencia entre ambas medidas. Además, solamente 7 individuos $(4,6 \%$ del total de la muestra) presentaron puntajes totales que traspasaron los límites de concordancia entre IDM-a e IDM-n.

Adicionalmente, la concordancia del puntaje asociado a cada uno de los 14 grupos alimentarios que forman IDM-Chile -cuando fue autoaplicado o aplicado por nutricionista- se evaluó mediante el coeficiente kappa ${ }^{22}$. La concordancia de todos los ítems del instrumento fue satisfactoria, con 7 grupos de alimentos mostrando acuerdo "casi 

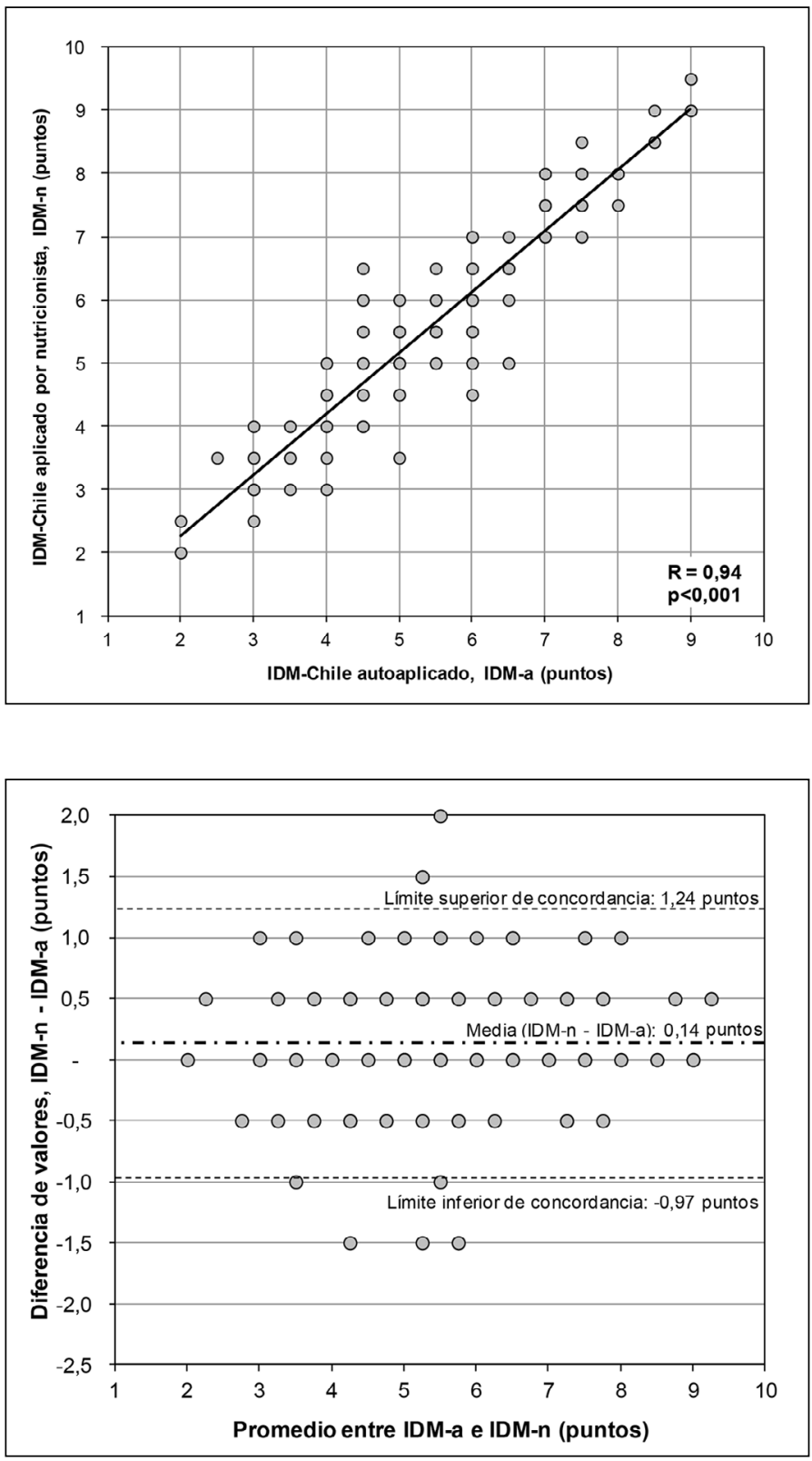

Figura 1. Correlación entre los puntajes del IDM-Chile autoaplicado (IDM-a) y el IDM-Chile aplicado por nutricionista (IDM-n) en 153 adultos chilenos. Cada punto de este gráfico puede representar a 1 o más individuos de la muestra de estudio dado que los puntajes del índice no son valores continuos.
Figura 2. Análisis de Bland-Altman para concordancia entre el IDM-Chile autoaplicado (IDM-a) y el IDM-Chile aplicado por nutricionista (IDM-n) en 153 adultos chilenos. Cada punto de este gráfico puede representar a 1 o más individuos de la muestra de estudio dado que los puntajes del índice no son valores continuos. perfecto" (kappa $>0,81$ ) y 7 grupos de alimentos con acuerdo sustancial (kappa $>0,61$ ), siendo el ítem de consumo de carnes rojas grasas y carnes procesadas el que tuvo menor concordancia (kappa $=0,694)$.

En resumen, la autoaplicación del IDM-Chile es válida con muy buen grado de asociación y concordancia de las respuestas obtenidas de esta forma con respecto a aquellas resultantes del mismo cuestionario aplicado por una nutricionista.

\section{Autoaplicación del IDM-Chile en población chilena adulta}

Las características demográficas, de estilo de vida y estado nutricional de la muestra de población chilena adulta estudiada se muestra en la Tabla 2. 
A

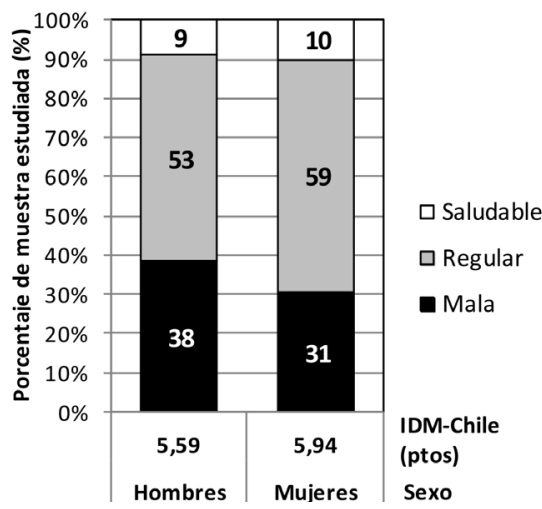

B

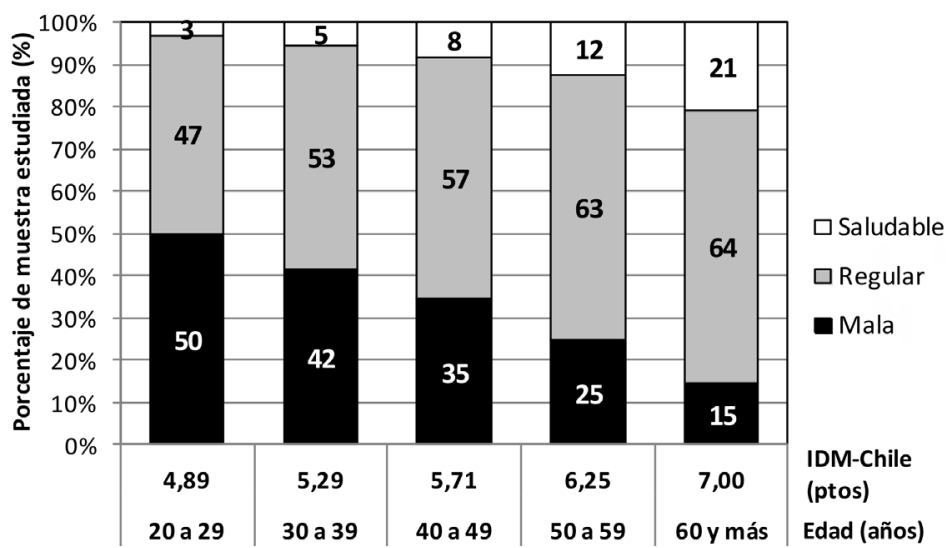

C

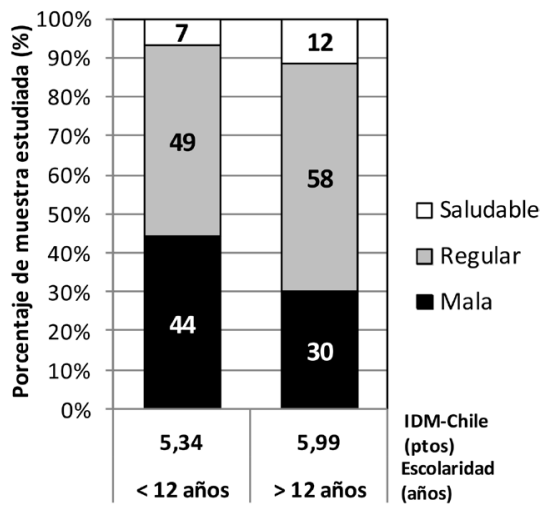

Figura 3. Variación del IDM-Chile según sexo (A), edad (B) y nivel educacional (C) en 53.366 adultos chilenos. Los datos fueron ajustados a la población chilena general por sexo, edad y región de residencia.

La evaluación de la adherencia a una dieta mediterránea mediante la autoaplicación del IDM-Chile mostró que solamente $9,5 \%$ de la muestra estudiada presentaba una alimentación categorizable como saludable y que las mujeres alcanzaron un puntaje promedio más alto que los hombres $(5,94$ vs 5,59; $\mathrm{p}<0,001)$ (Figura 3A). La adherencia a la dieta mediterránea mejoró con la edad, con un puntaje promedio que varió desde un mínimo de 4,9 en el rango de 20 a 29 años de edad hasta un máximo de 7,0 puntos en los sujetos mayores de 60 años $(\mathrm{p}<0,001)$ (Figura $3 \mathrm{~B})$. Adicionalmente, se observó un mejor índice de calidad alimentaria a mayor nivel educacional, 
Índice chileno de dieta mediterránea - G. Echeverría et al

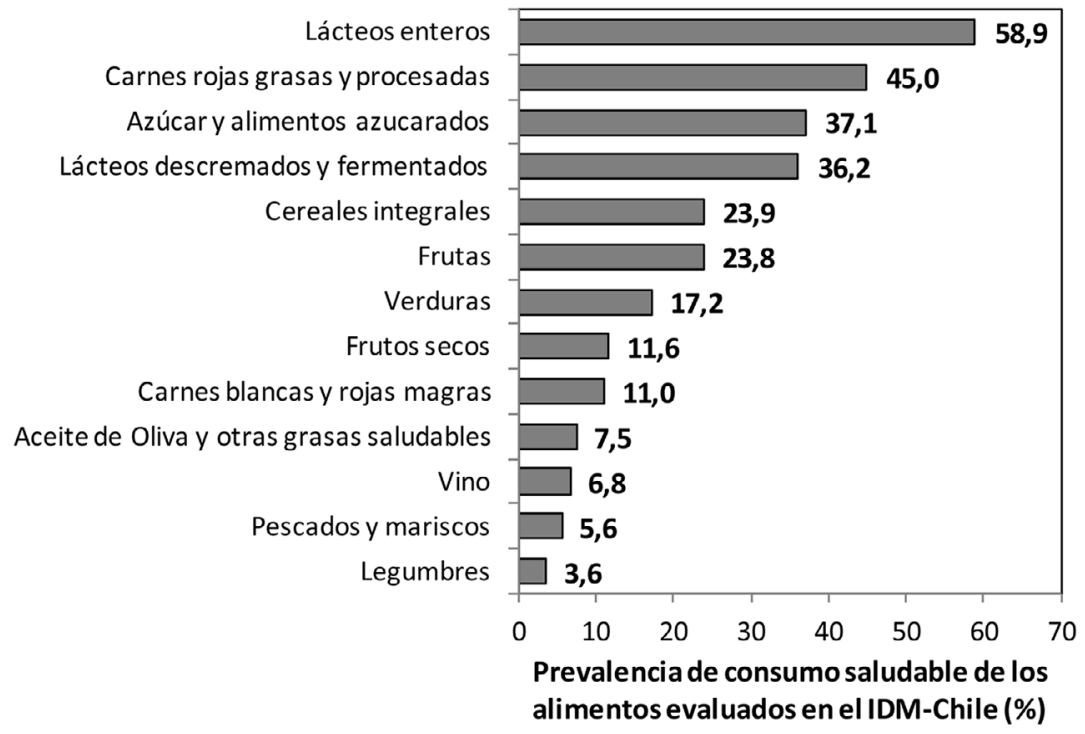

Figura 4. Prevalencia de consumo saludable de los grupos de alimentos evaluados por el IDM-Chile en 53.366 adultos chilenos. Un consumo saludable de cada grupo alimentario corresponde a un puntaje $=1$, excepto para aceite de oliva y otras grasas saludables cuyo puntaje óptimo es 2 . Los datos fueron ajustados a la población chilena general por sexo, edad y región de residencia. Consumos saludables o recomendados según IDM-Chile: lácteos enteros: no consume diariamente; carnes rojas grasas y procesadas: < 1 vez/semana; azúcar: < 4 cucharaditas/día; alimentos azucarados: no consume diariamente; lácteos descremados y fermentados: consumo diario; cereales integrales: $\geq 2$ porciones/día; frutas: $\geq 2$ porciones/día; verduras: $\geq 3$ porciones/día; frutos secos: $>2$ puñados/semana; carnes blancas y rojas magras: 5-8 veces/semana; aceite de oliva y otras grasas saludables: $\geq 3$ cucharaditas/día de aceite de oliva; vino: 1-2 copas/día y $\geq 4$ días/semana; pescados y mariscos: $>2$ veces/semana; y legumbres: $>2$ veces/semana.

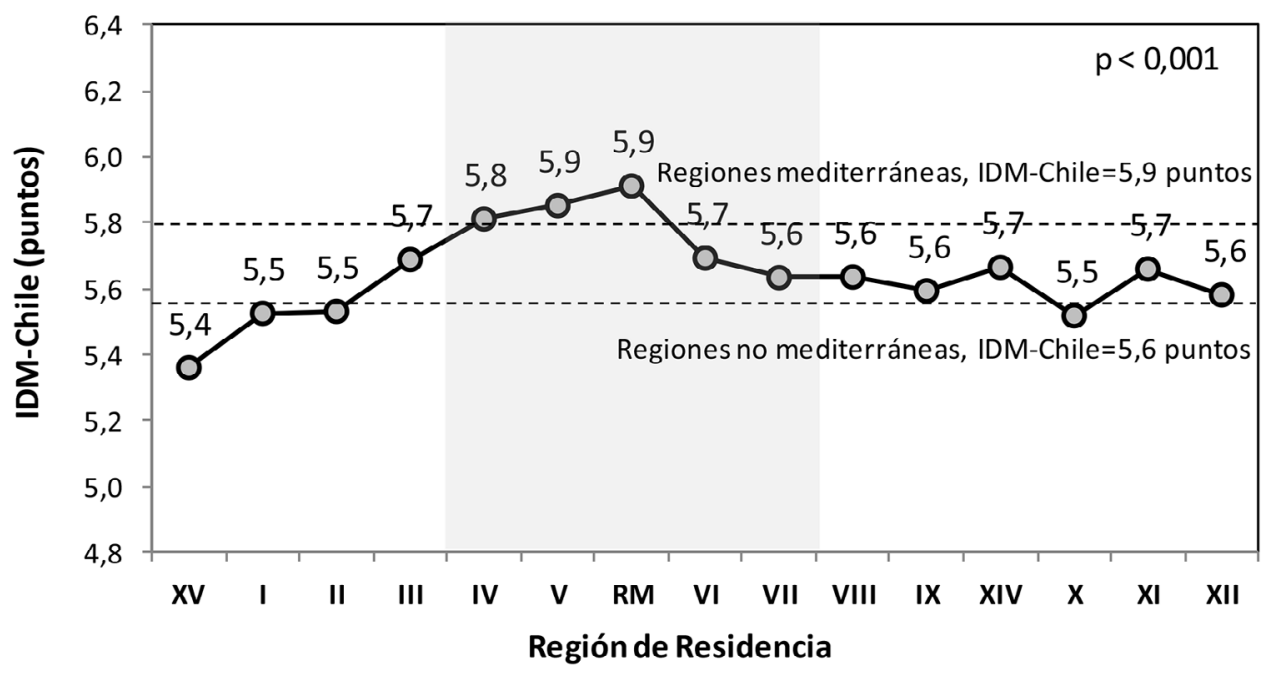

Figura 5. Variación del IDM-Chile según región de residencia en 53.366 adultos chilenos. Datos ajustados a población chilena general por sexo, edad y región de residencia. 
con un puntaje de 5,3 en el nivel educacional medio-bajo versus un puntaje de 6,0 en el nivel educacional alto $(\mathrm{p}<0,001)$ (Figura 3C).

Por otro lado, el consumo saludable o recomendado de cada alimento del índice (Tabla 1) fue mayor para los lácteos enteros, con 58,9\% de la población presentando una ingesta adecuada, seguido por el consumo de carnes rojas o procesadas con $45,0 \%$ y azúcar o alimentos azucarados con $37,1 \%$, lo cual corresponde a una ingesta baja de estos grupos de alimentos. Por el contrario, las recomendaciones de consumo saludable (ingesta alta) de aceite de oliva y otras grasas saludables, vino, pescados y mariscos y legumbres fueron las menos prevalentes, alcanzando 7,$5 ; 6,8 ; 5,6$ y $3,6 \%$, respectivamente (Figura 4 ).

Finalmente, el nivel de adherencia a la dieta mediterránea fue mayor en las zonas mediterráneas -IV a VII región- de Chile, mientras que el índice alcanzó su menor valor en las regiones más extremas del país (Figura 5).

\section{Discusión}

Los resultados de este estudio muestran que el IDM-Chile diseñado para nuestra población es un instrumento que puede ser autoaplicado válidamente -sin necesidad de un especialista- para evaluar la composición de la alimentación y el nivel de adherencia al patrón de dieta mediterránea.

La dieta mediterránea aporta elevadas cantidades de nutrientes, antioxidantes y otros compuestos bioactivos con numerosos beneficios para la salud ${ }^{23}$. Este patrón dietario ha ganado relevancia, ya que estudios epidemiológicos y de intervención lo han asociado o han demostrado que determina una baja incidencia de síndrome metabólico, diabetes, enfermedad cardiovascular, cáncer y enfermedades neurodegenerativas ${ }^{10-19,21}$. El primer índice desarrollado para medir adherencia a este patrón dietario fue el Mediterranean Diet Score (MDS) creado por Trichopoulou et $\mathrm{al}^{10}$. Este score ha sido posteriormente modificado y adaptado a diferentes poblaciones o países, existiendo hasta la fecha más de 20 índices mediterráneos publicados ${ }^{11}$.

La zona central de Chile corresponde a uno de los cinco ecosistemas del mundo con clima y, por tanto, producción agrícola semejante a los países de la cuenca del mediterráneo ${ }^{23-25}$. El presente índice, derivado del estudio de Leighton et al. ${ }^{20}$, con- sidera los alimentos característicos de esta dieta, incorporando otros propios de nuestro país, como la palta o aguacate, por su elevado contenido de ácidos grasos monoinsaturados. A su vez, el índice incluye tanto alimentos con conocidos beneficios para la salud (como frutas, verduras, legumbres, cereales integrales, pescado, frutos secos y aceite de oliva) e incluidos en otros índices mediterráneos, así como otros con efectos perjudiciales, como carnes grasas, azúcar y productos azucarados ${ }^{11}$. A diferencia de otros índices de este tipo, IDM-Chile distingue entre lácteos descremados y fermentados versus lácteos enteros, y además desagrega el consumo de carnes rojas entre cortes grasos versus magros ${ }^{11}$. Finalmente, nuestro índice considera la calidad de las grasas consumidas mediante la evaluación del consumo de aceites saludables como oliva y canola versus mantequilla, crema y lácteos enteros como fuentes de grasas saturadas perjudiciales para la salud.

La autoaplicación de este índice en una importante muestra de la población chilena $(>53.000$ sujetos) mostró tendencias similares -en cuanto a la calidad global de la alimentación- en comparación con otros índices como el Healthy Eating Index (HEI) de Estados Unidos de Norteamérica ${ }^{26}$ $\mathrm{u}$ otros índices aplicados en población española ${ }^{27}$ y chilena ${ }^{28}$. Usando estos índices en estas diferentes poblaciones, y concordante con nuestros hallazgos, se ha observado que la composición de la dieta es de mejor calidad en mujeres, a mayor edad y en niveles educacionales o socioeconómicos altos. Además, nuestro estudio demostró una diferencia significativa en el nivel de adherencia a la dieta mediterránea según la región de residencia, arrojando mayor puntaje en las zonas de nuestro país con un clima y producción agrícola semejante a los países mediterráneos. Aunque la muestra estudiada no es representativa de la población chilena general -ya que corresponden a usuarios de la plataforma online del Programa Aliméntate Sano-, estos resultados sugieren que IDM-Chile es un método adecuado para describir las características de su alimentación y el nivel de adherencia al patrón de dieta mediterránea. Sin embargo, es importante considerar que la población evaluada en este estudio tiene un alto nivel de escolaridad (72,9\% con más de 12 años de escolaridad), por lo que la aplicabilidad y la validez de este índice en poblaciones con menores o niveles más variados de educación requieren ser evaluadas en futuros 
estudios que cuenten con mayor representatividad de la población chilena, como ocurre en la Encuesta Nacional de Salud. Por otro lado, ciertos condicionantes sociales, como la pobreza o menores niveles educacionales de ciertos segmentos de nuestro país, podrían constituir potenciales barreras para una buena adherencia a la dieta mediterránea, dificultando la adquisición de ciertos alimentos de mayor costo -como el pescado o el aceite de oliva- y un acercamiento integral a este estilo de alimentación. Estos condicionantes sociales deben ser evaluados y considerados al momento de desarrollar estrategias que fomenten este patrón alimentario en la población chilena.

Como conclusión, la validación y el uso de este índice chileno de dieta mediterránea (IDM-Chile) breve, simple y autoaplicable muestra su utilidad para caracterizar la calidad de la alimentación en la población chilena, con tendencias demográficas comparables a otros índices internacionales. Sin embargo, estudios adicionales deberán establecer su asociación y valor predictivo del riesgo de enfermedades crónicas en nuestro país. Siendo la dieta mediterránea un patrón dietario con beneficio demostrado para la salud, este índice representaría una herramienta conveniente para la descripción general de la calidad de la dieta en la población chilena, entregando información fundamental para el desarrollo y seguimiento de estrategias orientadas a mejorar los aspectos deficientes de nuestra alimentación y disminuir la prevalencia de enfermedades crónicas en Chile.

Agradecimientos. La investigación ha sido financiada con aportes de Fundación Banmédica y Fundación Alimenta.

\section{Anexo 1. Cuestionario nutricional para cálculo del índice chileno de dieta mediterránea (IDM-Chile)}

1. ¿Cuántas porciones de verduras consume al día? Considere todo tipo de verduras, crudas o cocidas, consumidas como ensaladas, guisos, sopas (hechas de verdura natural), y/o acompañamientos calientes. Las papas no se consideran en este ítem.

1 porción de verduras: 1 taza o 1 plato de entrada de verduras

2 porciones de verduras: 2 tazas o 1 plato de fondo lleno de verduras

Ninguna o menos de 1

1 a 3 porciones

3 o más porciones

2. ¿Cuántas veces a la semana, en promedio, consume un plato de legumbres? Considere lentejas, garbanzos, porotos, arvejas secas o deshidratadas.

Ninguna o menos de 1

1 a 2 veces

más de 2 veces

3. ¿Cuántas veces a la semana, en promedio, consume un puñado de frutos secos? Considere nueces, almendras, maní, avellanas, castañas de cajú, pistachos, etc.

Ninguna o menos de 1

1 a 2 veces

más de 2 veces

4. ¿Cuántas porciones de frutas consume al día? Considere todo tipo de frutas, crudas, cocidas o deshidratadas (huesillos, pasas, higos secos, otros).

1 porción de frutas: 1 fruta grande (manzana, pera, naranja, plátano, durazno), 2 frutas chicas (kiwis, mandarinas),

1 taza de fruta picada

Ninguna o menos de 1

1 a 2 porciones

2 o más porciones 
5. ¿Cuántas porciones de cereales integrales consume regularmente cada día? Considere arroz y pasta integral, pan integral, otros cereales integrales como quínoa y avena, cereales integrales para el desayuno, galletas o galletones integrales, y todo tipo de masas o platos preparados a base de cereales integrales.

1 porción: 1 taza de pasta o arroz integral listo para servir, 1 taza de cereales integrales para el desayuno, 2 rebanadas (o 1 marraqueta o 1 hallulla) de pan integral

Ninguna o menos de 1

- 1 a 2 porciones

— 2 o más porciones

6. ¿Cuántas veces a la semana, en promedio, consume carnes con poca grasa? Considere carne de ave, pavo (jamón de pavo), cerdo magro (filete de cerdo, pulpa de cerdo, lomo de cerdo) y carnes rojas magras (posta, filete, palanca, lomo liso desgrasado, pollo ganso, asiento).

ninguna

1 a 4 veces

5 a 8 veces

más de 8 veces

7. ¿Cuántas veces a la semana, en promedio, consume carnes altas en grasa o procesadas? Considere carne de cerdo grasa (chuletas, costillas), carne roja grasa (lomo vetado, costillas), cordero, tocino, jamón, cecinas y embutidos, entrañas, hamburguesas preelaboradas.

_ ninguna o menos de 1

1 a 2 veces

más de 2 veces

8. ¿Cuántas veces a la semana, en promedio, consume pescados o mariscos?

ninguna o menos de 1

$$
1 \text { a } 2 \text { veces }
$$

más de 2

9.1. ¿Consume algún producto lácteo semidescremado, descremado o fermentado al día? Considere leche descremada y leche semidescremada, todo tipo de yogurt, todo tipo de leche cultivada, quesillo, queso fresco y quesos.

- Sí

Si la respuesta es NO, pasar directamente a la pregunta 10.1

9.2. ¿Cuántas tazas de lácteos descremados, semidescremados o fermentados consume al día? Considere leche descremada y leche semidescremada, todo tipo de yogurt, y todo tipo de leche cultivada.
no consumo
1 o menos tazas
más de 1 taza

10.1. ¿Consume diariamente leche entera, mantequilla o crema?

Sí

No

Si la respuesta es no, pasar directamente a la pregunta 11.1

10.2. ¿Consume regularmente más de 1 taza de leche entera al día?

Sí

No

10.3. ¿Consume regularmente más de 2 cucharaditas (de té) de mantequilla o crema al día?

Sí

No 
Índice chileno de dieta mediterránea - G. Echeverría et al

11.1. ¿Consume regularmente aceite de oliva?

No

Si la respuesta es no, pasar directamente a la pregunta 12.1

11.2. ¿Cuántas cucharaditas (de té) de aceite de oliva consume al día?

Ninguna

1 a 2 cucharaditas

3 o más cucharaditas

12.1. ¿Usa regularmente aceite canola puro en su casa? La pregunta se refiere a aceite canola no mezclado con otros aceites, como a veces está disponible en el comercio.

Sí

No

Ocasionalmente

No sabe / no lo conoce

12.2. ¿Cuántas paltas consume a la semana regularmente?

ninguna o menos de $1 / 2$ palta

$1 / 2$ a 3 paltas

_ más de 3 paltas

13.1. ¿Consume regularmente vino con las comidas (4 o más veces a la semana)?

No

Si la respuesta es no, pasar directamente a la pregunta 14.1

13.2. ¿Cuántas copas de vino consume al día en promedio?

menos de 1 copa

1 a 2 copas

más de 2 copas

14.1. ¿Consume regularmente dulces, golosinas o postres con azúcar más de 1 vez al día? Considere dulces, jalea normal (con azúcar), golosinas, chocolates, pasteles, tortas, queques, galletas azucaradas, etc.

Sí

No

14.2. ¿Consume habitualmente bebidas gaseosas o jugos no light o azucarados durante el día?

Sí

No

14.3. En promedio, ¿cuántas cucharaditas (de té) de azúcar consume al día?

menos de 4 cucharaditas

4 o más cucharaditas

Ninguna o endulzantes (sacarina, aspartame, sucralosa, estevia, otro) 


\section{Anexo 2. Algoritmo de cálculo de puntaje del índice chileno de dieta mediterránea (IDM-Chile)}

El puntaje total del IDM-Chile se obtiene por la suma aritmética de los puntajes de cada uno de los ítems evaluados.

Puntaje IDM-Chile: Verd + Leg + FSecos + Frut + Cerlnt + CMagra + CGrasa + Pescado + LacDesc + LacEnt + Oliva_GSaludables + Vino + Azúcar

\begin{tabular}{|c|c|c|c|c|}
\hline & \multirow{2}{*}{$\begin{array}{c}\mathbf{N}^{\circ} \text { de } \\
\text { pregunta }\end{array}$} & \multicolumn{3}{|c|}{ Obtención del puntaje de cada ítem } \\
\hline & & 0 ptos & 0,5 ptos & 1 pto \\
\hline Verd & 1. & P1., alt.1 & P1., alt.2 & P1., alt.3 \\
\hline Leg & 2. & P2., alt.1 & P2., alt.2 & P2., alt.3 \\
\hline FSecos & 3. & P3., alt.1 & P3., alt.2 & P3., alt.3 \\
\hline Frut & 4. & P4., alt.1 & P4., alt.2 & P4., alt.3 \\
\hline Cerlnt & 5. & P5., alt.1 & P5., alt.2 & P5., alt.3 \\
\hline CMagra & 6. & P6., alt.1 o P6., alt.4 & P6., alt.2 & P6., alt.3 \\
\hline CGrasa & 7. & P7., alt.3 & P7., alt.2 & P7., alt.1 \\
\hline Pescado & 8. & P8., alt.1 & P8., alt.2 & P8., alt.3 \\
\hline LacDesc & 9. & (P9.1., alt.2) & $\begin{array}{l}\text { (P9.1., alt.1 y P9.2., alt.1), o } \\
\text { (P9.1., alt.1 y P9.2., alt.2) }\end{array}$ & P9.1., alt.1 y P9.2., alt.3 \\
\hline LacEnt & $\begin{array}{c}10.1 \mathrm{y} \\
10.2\end{array}$ & $\begin{array}{l}\text { (P10.1., alt.1 y P10.2., alt. } 1 \text { y } \\
\text { P10.3., alt.1), o (P10.1., alt. } 1 \text { y } \\
\text { P10.2., alt. } 1 \text { y P10.3., alt.2), o } \\
\text { (P10.1., alt.1 y P10.2., alt.2 y } \\
\text { P10.3., alt.1) }\end{array}$ & $\begin{array}{l}\text { (P10.1., alt.1 y P10.2., alt.2 y } \\
\text { P10.3., alt.2) }\end{array}$ & P10.1., alt.2 \\
\hline Vino & $\begin{array}{l}13.1 \mathrm{y} \\
13.2 \\
\end{array}$ & P13.1., alt.2 & $\begin{array}{l}\text { (P13.1., alt.1 y P13.2., alt.1), o } \\
\text { (P13.1., alt.1 y P13.2., alt.3) }\end{array}$ & (P13.1., alt.1 y P13.2., alt.2) \\
\hline Azúcar & $\begin{array}{c}14.1,14.2 \\
y \\
14.3\end{array}$ & $\begin{array}{l}\text { (P14.1., alt.1 y P14.2., alt.1 y } \\
\text { P14.3., alt.1), o (P14.1., alt. } 1 \text { y } \\
\text { P14.2., alt. } 1 \text { y P14.3., alt.2), o } \\
\text { (P14.1., alt.1 y P14.2., alt. } 1 \text { y } \\
\text { P14.3., alt.3), o (P14.1., alt.1 y } \\
\text { P14.2., alt.2 y P14.3., alt.2) }\end{array}$ & $\begin{array}{l}\text { (P14.1., alt.1 y P14.2., alt.2 y } \\
\text { P14.3., alt.1), o (P14.1., alt.1 y } \\
\text { P14.2., alt.2 y P14.3., alt.3), o } \\
\text { (P14.1., alt.2 y P14.2., alt.1 y } \\
\text { P14.3., alt.1), o (P14.1., alt.2 y } \\
\text { P14.2., alt.1 y P14.3., alt.2), o } \\
\text { (P14.1., alt.2 y P14.2., alt.1 y } \\
\text { P14.3., alt.3), o (P14.1., alt.2 y } \\
\text { P14.2., alt.2 y P14.3., alt.2) }\end{array}$ & $\begin{array}{l}\text { (P14.1., alt.2 y P14.2., alt.2 y } \\
\text { P14.3., alt.1), o (P14.1., alt.2 y } \\
\text { P14.2., alt.2 y P14.3., alt.3) }\end{array}$ \\
\hline
\end{tabular}

El puntaje del ítem de aceite de oliva y otras grasas saludables (Oliva_GSaludables) puede tomar valores de $0 ; 0,5 ; 1 ; 1,5$ y 2 puntos, y se compone de 2 ítems evaluados en el IDM-Chile: consumo de aceite de oliva (preguntas 11.1 y 11.2 ) y consumo de aceite Canola y palta (preguntas 12.1 y 12.2), según el siguiente algoritmo de cálculo:

0 pto: Oliva $=0$ y (Palta + Canola $)<1$

0,5 ptos: $($ Oliva $=0,5$ y $($ Palta + Canola $)<1)$ o $($ Oliva $=0$ y $($ Palta + Canola $)=1)$

1 pto: $($ Oliva $=0,5$ y $($ Palta + Canola $)=1)$ o $($ Oliva $=0$ y $($ Palta + Canola $) \geq 1,5)$

1,5 ptos: Oliva $=0,5$ y (Canola + Palta $) \geq 1,5$

2 ptos: Oliva $=1$

Donde:

\begin{tabular}{|l|c|l|l|l|}
\hline & \multirow{2}{*}{$\begin{array}{c}\mathbf{N}^{\circ} \text { de } \\
\text { pregunta }\end{array}$} & Obtención del puntaje de cada ítem \\
\cline { 3 - 5 } & & $\mathbf{0}$ ptos & $\mathbf{0 , 5}$ ptos & $\mathbf{1}$ pto \\
\hline Oliva & $11,1 \mathrm{y}$ & $\mathrm{P} 11,1 .$, alt.2 & $\begin{array}{l}(\mathrm{P} 11,1 ., \text { alt.1 y } \mathrm{P} 11,2 ., \text { alt.1), o } \\
(\mathrm{P} 11,1 ., \text { alt.1 y } \mathrm{P} 11,2 ., \text { alt.2 })\end{array}$ & $(\mathrm{P} 11,1 .$, alt.1 y $\mathrm{P} 11,2 .$, alt.3) \\
\hline Canola & 12,1 & $\mathrm{P} 12,1 .$, alt.2 o $\mathrm{P} 12,1 .$, alt.4 & $\mathrm{P} 12,1 .$, alt.3 & $\mathrm{P} 12,1 .$, alt.1 \\
\hline Palta & 12,2 & $\mathrm{P} 12,2 .$, alt.1 & $\mathrm{P} 12,2 .$, alt.2 & $\mathrm{P} 12,2 .$, alt.3 \\
\hline
\end{tabular}




\section{Referencias}

1. Hu FB. Dietary pattern analysis: a new direction in nutritional epidemiology. Curr Opin Lipidol 2002; 13 (1): 3-9.

2. Mozaffarian D, Ludwig DS. Dietary guidelines in the $21 \mathrm{st}$ century-a time for food. JAMA 2010; 304 (6): 681-2.

3. Gil A, Martínez de Victoria E, Olza J. Indicators for the evaluation of diet quality. Nutr Hosp 2015; 31 Suppl 3: 128-44.

4. Waijers PM, Feskens EJ, Ocke MC. A critical review of predefined diet quality scores. Br J Nutr 2007; 97 (2): 219-31.

5. Kant AK. Dietary patterns: biomarkers and chronic disease risk. Appl Physiol Nutr Metab 2010; 35 (2): 199206.

6. Harmon BE, Boushey CJ, Shvetsov YB, Ettienne R, Reedy J, Wilkens LR, et al. Associations of key diet-quality indexes with mortality in the Multiethnic Cohort: the Dietary Patterns Methods Project. Am J Clin Nutr 2015; 101 (3): 587-97.

7. Kennedy ET, Ohls J, Carlson S, Fleming K. The Healthy Eating Index: design and applications. J Am Diet Assoc 1995; 95 (10): 1103-8.

8. Patterson RE, Haines PS, Popkin BM. Diet quality index: capturing a multidimensional behavior. J Am Diet Assoc 1994; 94 (1): 57-64.

9. Huijbregts P, Feskens E, Räsänen L, Fidanza F, Nissinen A, Menotti A, Kromhout D. Dietary pattern and 20 year mortality in elderly men in Finland, Italy, and The Netherlands: longitudinal cohort study. BMJ 1997; 315 (7099): 13-7.

10. Trichopoulou A, Kouris-Blazos A, Wahlqvist ML, Gnardellis C, Lagiou P, Polychronopoulos E, et al. Diet and overall survival in elderly people. BMJ 1995; 311 (7018): 1457-60.

11. Hernández-Ruiz A, García-Villanova B, Guerra Hernández EJ, Amiano $\mathrm{P}$, Azpiri $\mathrm{M}$, Molina-Montes $\mathrm{E}$. Description of indexes based on the adherence to the Mediterranean dietary pattern: a review. Nutr Hosp 2015; 32 (5): 1872-84.

12. García-Fernández E, Rico-Cabanas L, Rosgaard N, Estruch R, Bach-Faig A. Mediterranean diet and cardiodiabesity: a review. Nutrients 2014; 6 (9): 3474-500.

13. Dussaillant C, Echeverría G, Urquiaga I, Velasco N, Rigotti A. Evidencia actual sobre los beneficios de la dieta mediterránea en salud. Rev Med Chile 2016; 144 (8): 1044-52.

14. Alberti-Fidanza A, Fidanza F. Mediterranean Adequacy Index of Italian diets. Public Health Nutr 2004; 7 (7): 937-41.

15. Martínez-González MA, Fernández-Jarne E, Serrano-Martínez M, Wright M, Gómez-Gracia E. Development of a short dietary intake questionnaire for the quantitative estimation of adherence to a cardioprotective Mediterranean diet. Eur J Clin Nutr 2004; 58 (11): 1550-2.

16. Sofi F, Abbate R, Gensini GF, Casini A. Accruing evidence on benefits of adherence to the Mediterranean diet on health: an updated systematic review and meta-analysis. Am J Clin Nutr 2010; 92 (5): 1189-96.

17. Trichopoulou A, Bamia C, Trichopoulos D. Anatomy of health effects of Mediterranean diet: Greek EPIC prospective cohort study. BMJ 2009; 338: b2337.

18. Buckland G, Agudo A, Travier N, Huerta JM, Cirera L, Tormo MJ, et al. Adherence to the Mediterranean diet reduces mortality in the Spanish cohort of the European Prospective Investigation into Cancer and Nutrition (EPIC-Spain). Br J Nutr 2011; 106 (10): 1581-91.

19. Knoops KT, de Groot LC, Kromhout D, Perrin AE, Moreiras-Varela O, Menotti A, et al. Mediterranean diet, lifestyle factors, and 10-year mortality in elderly European men and women: the HALE project. JAMA 2004; 292 (12): 1433-9.

20. Leighton F, Polic G, Strobel P, Pérez D, Martínez C, Vásquez L, et al. Health impact of Mediterranean diets in food at work. Public Health Nutr 2009; 12 (9A): 1635-43.

21. Salas-Salvado J, Fernández-Ballart J, Ros E, Martínez-González MA, Fito M, Estruch R, et al. Effect of a Mediterranean diet supplemented with nuts on metabolic syndrome status: one-year results of the PREDIMED randomized trial. Arch Intern Med 2008; 168 (22): 2449-58.

22. Landis JR, Koch GG. The measurement of observer agreement for categorical data. Biometrics 1977; 33 (1): 159-74.

23. Urquiaga I, Echeverría G, Dussaillant C, Rigotti A. Origen, Componentes y Posibles Mecanismos de Acción de la Dieta Mediterránea Rev Med de Chile. 2016; Artículo en prensa.

24. Köppen V. Das geographische System der Klimate. Köppen V, Geiger R, editors. Berlin: Gebrüder Borntraeger; 1936.

25. Groves RH. Biogeography of Mediterranean invasions. Groves RH, di-Castri F, editors. Cambridge: Cambridge University Press; 1992.

26. Ervin RB. Healthy Eating Index-2005 total and component scores for adults aged 20 and over: National Health and Nutrition Examination Survey, 2003-2004. Natl Health Stat Report 2011 (44): 1-9.

27. Norte Navarro AI, Ortiz Moncada R. [Spanish diet quality according to the healthy eating index]. Nutr Hosp 2011; 26 (2): 330-6.

28. Dussaillant C, Echeverría G, Villarroel L, Marín PP, Rigotti A. [Unhealthy Food Intake Is Linked to Higher Prevalence of Metabolic Syndrome in Chilean Adult Population: Cross Sectional Study in 2009-2010 National Health Survey]. Nutr Hosp 2015; 32 (5): 2098-104. 